\title{
Resposta germinativa de duas espécies de Vellozia (Velloziaceae) dos campos rupestres de Minas Gerais, Brasil
}

\author{
Queila de Souza Garcia ${ }^{1,3}$, Claudia Maria Jacobi² e Beatriz de Aquino Ribeiro²
}

Recebido em 27/01/2006. Aceito em 9/11/2006

\begin{abstract}
RESUMO - (Resposta germinativa de duas espécies de Vellozia (Velloziaceae) dos campos rupestres de Minas Gerais, Brasil). Foi avaliado o comportamento germinativo das sementes de Vellozia leptopetala Goeth. et Henr., uma espécie de porte arbustivo que cresce sobre afloramentos rochosos e de V. epidendroides Mart. ex Schult. \& Schult., uma herbácea que cresce em campos, na Serra do Cipó. As sementes foram submetidas às temperaturas de 15 a $40{ }^{\circ} \mathrm{C}$, sob luz ou escuro contínuos, com quatro repetições de 25 sementes por tratamento. Ambas as espécies apresentam sementes pequenas, com 1,31 0,06 mm e 1,15 0,01 mm para V. leptopetala e V. epidendroides, respectivamente. As sementes de V. leptopetala sob luz apresentaram germinabilidade alta $(95$ a $100 \%)$ e semelhante na faixa de 20 a 35 ${ }^{\circ} \mathrm{C}$ e percentuais inferiores a 15 e $40{ }^{\circ} \mathrm{C}(34$ e $29 \%$, respectivamente). Na condição de escuro a germinação só ocorreu nas temperaturas mais altas, alcançando $68 \%$ a $30{ }^{\circ} \mathrm{C}$ e $3 \%$ a $40{ }^{\circ} \mathrm{C}$. Na presença de luz as sementes de $V$. epidendroides apresentaram altos percentuais de germinação na faixa de 30 a $40{ }^{\circ} \mathrm{C}(91$ a $84 \%)$, com diminuição gradativa da germinabilidade nas temperaturas mais baixas e inibição do processo a $15{ }^{\circ} \mathrm{C}$. No escuro ocorreu germinação na faixa de 20 a $40{ }^{\circ} \mathrm{C}\left(3\right.$ a $88 \%$ ), sendo significativamente superior a 35 e $40{ }^{\circ} \mathrm{C}$. A produção de sementes capazes de germinar em diferentes condições de temperatura e luminosidade pode ser de grande valor para a espécie, por permitir a colonização de maior diversidade de hábitats, como fendas de rochas sem luz, situação comum para V. leptopetala, ou com densa cobertura por herbáceas, para V. epidendroides.
\end{abstract}

Palavras-chave: fotoblastismo, temperatura, campos rupestres, Vellozia leptopetala, Vellozia epidendroides

\begin{abstract}
Germination response of two species of Vellozia (Velloziaceae) from the "campos rupestres" of Minas Gerais, Brazil). The germination behaviour of Vellozia leptopetala Goeth. et Henr., a shrubby species growing on rocky outcrops, and of V. epidendroides Mart. ex Schult. \& Schult., an herb growing in grasslands of Serra do Cipó, was evaluated under controlled light and temperature conditions. Seeds were sown at temperatures ranging from 15 to $40{ }^{\circ} \mathrm{C}$ in continuous light or darkness, in four treatments with 25 seeds each. Both species produce small seeds; $1.31 \pm 0.06 \mathrm{~mm}$ and $1.15 \pm 0.01 \mathrm{~mm}$ for V. leptopetala and V. epidendroides, respectively. $V$. leptopetala seeds grown in light had high germinability (95-100\%) between 20 and $35^{\circ} \mathrm{C}$, and lower percentages at 15 and $40{ }^{\circ} \mathrm{C}(34$ and $29 \%$, respectively). When grown in total darkness, significant germination occurred only at the highest temperatures, reaching $68 \%$ at $30{ }^{\circ} \mathrm{C}$ and $3 \%$ at $40{ }^{\circ} \mathrm{C}$. V. epidendroides seeds grown in light had high germinability (91-84\%) between 30 and $40{ }^{\circ} \mathrm{C}$, which gradually decreased at lower temperatures to no germination at $15^{\circ} \mathrm{C}$. In darkness germination was successful between 20 and $40{ }^{\circ} \mathrm{C}(3$ to $88 \%)$, and was significantly higher at 35 and $40^{\circ} \mathrm{C}$. The production of seeds capable of germinating in different temperature and light conditions may be of great value to a species, because it allows colonization of a greater diversity of habitats, including dark rock crevices, a common situation for V. leptopetala, or thick grass cover, for $V$. epidendroides.
\end{abstract}

Key words: photoblastism, campos rupestres, temperature, Vellozia leptopetala, Vellozia epidendroides

\section{Introdução}

Diversos fatores podem afetar o sucesso reprodutivo de uma espécie, entre eles, o número e a qualidade das sementes produzidas (Stephenson 1981; Haig \& Westoby 1988). Uma vez que o valor adaptativo, ou fitness, de um organismo é definido como sua contribuição em número de indivíduos à geração seguinte na população, fatores que afetam a produção e a viabilidade das sementes estão entre os mais importantes a serem avaliados, principalmente em espécies endêmicas e/ou ameaçadas. A fase inicial da vida das plantas é considerada uma das mais cruciais, pois o estabelecimento de populações dependerá da

\footnotetext{
1 Universidade Federal de Minas Gerais, Instituto de Ciências Biológicas, Departamento de Botânica, C. Postal 486, $30161-970$ Belo Horizonte, MG, Brasil

2 Universidade Federal de Minas Gerais, Instituto de Ciências Biológicas, Departamento de Biologia Geral, C. Postal 486, $30161-970$ Belo Horizonte, MG, Brasil (jacobi@mono.icb.ufmg.br)

3 Autor para correspondência: queila@ netuno.lcc.ufmg.br
} 
capacidade de sementes e plântulas de lidar com condições ambientais adversas ou variáveis (Franco \& Silvertown 1997).

A qualidade das sementes também exerce influência sobre o sucesso reprodutivo das plantas. Características como tamanho, forma e textura podem afetar de forma diferente o potencial de sobrevivência dos juvenis e adultos, além da capacidade de dispersão da semente (Wulff 1995). Outro aspecto fundamental para o sucesso reprodutivo é a resposta germinativa às condições do meio. A adaptação às condições locais e plasticidade de resposta são qualidades necessárias, tanto para o estabelecimento efetivo dentro da população, como para ocupar novos ambientes ou enfrentar situações adversas (Venable \& Brown 1988).

Observações das respostas germinativas têm mostrado que o controle desse processo é exercido por fatores físicos do ambiente como luz e temperatura e a combinação destas condições influencia diretamente no estabelecimento das plântulas (Baskin \& Baskin 1988; Ghersa et al. 1992) e pode promover o aparecimento de características germinativas diversificadas entre as espécies vegetais (VázquezYanes \& Orozco-Segovia 1993). Assim, sementes com ampla capacidade de resposta a condições edafoclimáticas seriam as responsáveis por manter populações em ambientes que impõem uma série de restrições à colonização, incluindo-se escassez de solo, amplitudes térmicas elevadas, insolação, baixo teor de água ou prolongado déficit hídrico. Em ecossistemas como os campos de altitude, as plantas devem enfrentar o efeito combinado de vários destes fatores (Gaff 1987; Porembski \& Barthlott 2000).

A família Velloziaceae, de distribuição essencialmente tropical, tem o Brasil como centro principal de dispersão na América do Sul (Smith \& Ayensu 1976). As espécies desta família ocorrem principalmente nos campos rupestres, a altitudes entre 1.000 e $2.000 \mathrm{~m}$, especialmente nas formações quartzíticas da Cadeia do Espinhaço (Alves \& Kolbek 1994), onde é encontrada a grande maioria das mais de 140 espécies conhecidas do gênero Vellozia (MelloSilva 1995).

O objetivo deste trabalho foi avaliar o comportamento germinativo de duas espécies simpátricas de Vellozia, uma delas considerada ameaçada pela sua distribuição restrita (Mendonça \& Lins 2000). Foi analisada a resposta germinativa sob condições controladas de temperatura e luminosidade, associando este padrão de germinação às condições climáticas a que estão sujeitas suas populações.

\section{Material e métodos}

Área de estudo - A Serra do Cipó ocupa a porção intermediária entre os limites norte e sul da Cadeia do Espinhaço, distando cerca de $100 \mathrm{~km}$ a noroeste de Belo Horizonte. A região apresenta altitudes que variam entre 1.000 e $1.400 \mathrm{~m}$, atingindo até $1.800 \mathrm{~m}$ (Menezes \& Giulietti 1986). O clima é mesotérmico e denomina-se tropical de altitude, com verões brandos e alta precipitação nessa estação. A temperatura média anual oscila entre 17,4 e $19,8^{\circ} \mathrm{C}$ (Sendulsky \& Burman 1978) e a precipitação média anual é de $1.600 \mathrm{~mm}$, com inverno seco de três a quatro meses e um período úmido de sete a oito meses (Nimer 1989). O clima também é caracterizado por ventos fortes, insolação, variação térmica diária elevada e ocorrência de queimadas. Estes fatores promoveram uma convergência adaptativa, principalmente morfológica entre as espécies, para características xeromórficas (Giulietti \& Pirani 1988).

O tipo vegetacional predominante na Serra do Cipó, como em toda a Cadeia do Espinhaço, são os campos rupestres, sendo a flora desse ecossistema caracterizada pela alta diversidade e endemismo (Giulietti et al. 1987; Giulietti \& Pirani 1988). A vegetação rupestre da Serra do Cipó é constituída principalmente por um estrato herbáceo contínuo que ocupa os espaços entre os afloramentos rochosos, crescendo em solos rasos e arenosos, onde predominam representantes das famílias Poaceae, Cyperaceae e, tipicamente, Eriocaulaceae, Xyridaceae e Velloziaceae (Menezes \& Giulietti 1986). Onde o substrato é pedregoso, vêem-se arbustos e subarbustos isolados ou em pequenos grupos, representantes das famílias Velloziaceae, Asteraceae e Melastomataceae, entre outras. Nos afloramentos rochosos destacamse principalmente as Bromeliaceae, Cactaceae, várias espécies de Orchidadeae e Velloziaceae (Menezes \& Giulietti 1986).

Material biológico - Foram estudadas duas espécies de Vellozia que, apesar de simpátricas na Serra do Cipó, ocupam micro-hábitats diferentes. Vellozia leptopetala Goeth. et Henr. é uma espécie subarbustiva ramificada, atingindo $1,5 \mathrm{~m}$ de altura e floresce no início do verão. Os indivíduos crescem, principalmente, entre os afloramentos rochosos, sobre a camada fina de areia e/ou húmus que se acumula entre as pedras (Sazima \& Sazima 1990). Vellozia epidendroides Mart. ex Schult. \& Schult. tem porte herbáceo e ocorre em agrupamentos densos e discretos em áreas abertas de campos de altitude 
$(1.100-1.200 \mathrm{~m})$. As plantas adultas atingem $30-40 \mathrm{~cm}$ de altura e geralmente florescem no final do verão. As espécies são polinizadas por abelhas e, no caso de V. leptopetala, também por beija-flores (Sazima \& Sazima 1990; Jacobi \& del Sarto). Os frutos de ambas as espécies são cápsulas que podem conter várias centenas de pequenas sementes.

Metodologia - As sementes utilizadas neste estudo foram provenientes de 50 indivíduos de cada espécie, de populações localizadas na Serra do Cipó, MG $\left(19^{\circ} 17^{\prime} \mathrm{S}\right.$ e $\left.43^{\circ} 35^{\prime} \mathrm{W}\right)$. As sementes foram medidas com paquímetro digital $(n=100)$, sendo o comprimento considerado como a maior distância entre as extremidades (Garcia \& Diniz 2003). Os testes de germinação foram realizados em câmaras de germinação com luz e temperaturas controladas. Foram utilizadas temperaturas constantes entre 15 e $40{ }^{\circ} \mathrm{C}$, com intervalos de $5^{\circ} \mathrm{C}$, sob luz $\left(30 \mu \mathrm{mol} \mathrm{m}^{-2} \mathrm{~s}^{-1}\right)$ e escuro contínuos. As sementes foram colocadas para germinar em placas de Petri, forradas com folha dupla de papel de filtro e umedecidas com água destilada, utilizando-se quatro repetições de 25 sementes por tratamento. A condição de escuro contínuo foi obtida envolvendo-se as placas de Petri em papel alumínio e colocando-as em sacos pretos de polietileno. A observação das sementes sob escuro foi feita sob luz verde de segurança (Garcia \& Diniz 2003). As placas foram examinadas diariamente para contagem e remoção das sementes germinadas, até a estabilização da resposta, sendo o critério de germinação a protrusão da radícula.

Os dados de porcentagem, previamente transformados em valores angulares, e os de tempo médio de germinação foram submetidos a testes de normalidade (Shapiro-Wilk) e homogeneidade (BrownForsytle) e posteriormente à análise de variância (ANOVA) e comparados pelo teste de Tukey à 0,05 de significância. $O$ tempo médio de germinação foi calculado de acordo com Labouriau (1983) apenas para os tratamentos com germinabilidade superior a $10 \%$.

\section{Resultados e discussão}

Ambas as espécies apresentam sementes pequenas, com 1,31 $\pm 0,06 \mathrm{~mm}$ e 1,15 $\pm 0,01 \mathrm{~mm}$ para Vellozia leptopetala e V. epidendroides, respectivamente. $\mathrm{O}$ tamanho das sementes afeta muitos aspectos da ecologia das plantas (Moles et al. 2005), e tem relação direta com o comportamento germinativo (Leishmann et al. 2000) e a longevidade no solo (Thompson et al. 1993). Por possuírem recursos limitados, as sementes pequenas demandam requerimentos bem definidos para germinarem, entre os quais fotoblastismo e temperaturas específicas (Grime et al. 1981). Essas características influenciam diretamente o sucesso do estabelecimento da planta (Pearson et al. 2003) e foram registradas em várias espécies típicas de campos rupestres (Oliveira \& Garcia 2005; Abreu \& Garcia 2005).

As sementes de Vellozia leptopetala apresentaram germinabilidades altas e estatisticamente semelhantes na faixa de 20 a $35^{\circ} \mathrm{C}$, sob luz (Tab. 1). A 15 e a $40{ }^{\circ} \mathrm{C}$ a porcentagem final de germinação foi significativamente inferior à das demais temperaturas (aproximadamente um terço). O início da germinação ocorreu por volta do quinto dia na faixa de 25 a $35^{\circ} \mathrm{C}$, no sétimo dia a $40^{\circ} \mathrm{C}$, no nono dia a $20^{\circ} \mathrm{C}$ e após 20 dias de experimento a $15{ }^{\circ} \mathrm{C}$. Os tempos médios de germinação aumentaram nas temperaturas mais baixas, sendo superior a 40 dias na temperatura de $15^{\circ} \mathrm{C}$ (Tab. 1). As sementes responderam à condição de escuro contínuo apenas nas temperaturas de 30 e $35^{\circ} \mathrm{C}$, com germinabilidades próximas de $70 \%$ (Tab. 1). $\mathrm{O}$ início da germinação no escuro foi mais tardio do que na presença da luz, ocorrendo após nove dias a $30{ }^{\circ} \mathrm{C}$ e oito a $35^{\circ} \mathrm{C}$.

As sementes de Vellozia epidendroides apresentaram alto percentual de germinação na presença de luz, sem diferença significativa de 30 a $40{ }^{\circ} \mathrm{C}$ (Tab. 2). Sob temperaturas mais baixas a germinabilidade foi gradativamente reduzida, não ocorrendo germinação a $15{ }^{\circ} \mathrm{C}$. Sob escuro ocorreu germinação na faixa de 20 a $40{ }^{\circ} \mathrm{C}$, sendo muito baixa de 20 a $30^{\circ} \mathrm{C}$, variando de 3 a $14 \%$ e significativamente superior a 35 e a $40{ }^{\circ} \mathrm{C}$ (73 e $88 \%$, respectivamente). A germinação se iniciou no quinto dia na faixa de 25 a $40{ }^{\circ} \mathrm{C}$ e no nono dia a $20{ }^{\circ} \mathrm{C}$, tanto na luz como no escuro. $\mathrm{O}$ tempo médio de germinação sob luz variou de 10 a 12 dias na faixa de 25 a $40{ }^{\circ} \mathrm{C}$, sendo de 25 dias sob a temperatura de $15^{\circ} \mathrm{C}$ (Tab. 2). Na condição de escuro contínuo o tempo médio foi semelhante ao observado na presença da luz, de 30 a $40{ }^{\circ} \mathrm{C}$.

As veloziáceas apresentam diversas características de tolerância às condições edáficas e hídricas extremas, como as que ocorrem nos campos rupestres. $\mathrm{O}$ crescimento e a atividade fotossintética podem cessar nos períodos de déficit hídrico, motivo pelo qual foram consideradas plantas quiescentes por Owoseye \& Sanford (1972). Em espécies arborescentes do gênero Vellozia foi constatada a presença de um velamen radicum, que permite uma rápida absorção de água de chuva e da umidade do ar (Porembski \& 
454 Garcia, Jacobi \& Ribeiro: Resposta germinativa de duas espécies de Vellozia (Velloziaceae) dos campos rupestres...

Tabela 1. Germinabilidade final e tempo médio de germinação de sementes de Vellozia leptopetala Goeth. et Henr. (média \pm desvio padrão). Na coluna, valores seguidos por letras iguais não diferem estatisticamente entre si $(P>0,05)$. $W$ : estatística do teste de ShapiroWilk; $F$ : estatística do teste de Brown-Forsytle.

\begin{tabular}{cccccc}
\hline \multirow{2}{*}{ Temperatura $\left({ }^{\circ} \mathrm{C}\right)$} & \multicolumn{2}{c}{ Germinabilidade $(\%)$} & & \multicolumn{2}{c}{ Tempo médio de germinação (dias) } \\
\cline { 2 - 3 } \cline { 5 - 5 } & Luz & Escuro & & Luz & Escuro \\
\hline 15 & $34 \pm 14,8 \mathrm{~b}$ & $0 \mathrm{c}$ & & $42,2 \pm 2,6 \mathrm{a}$ & - \\
20 & $95 \pm 3,8 \mathrm{a}$ & $0 \mathrm{c}$ & & $19,0 \pm 1,8 \mathrm{~b}$ & - \\
25 & $98 \pm 4,0 \mathrm{a}$ & $0 \mathrm{c}$ & & $10,5 \pm 0,6 \mathrm{c}$ & - \\
30 & $96 \pm 3,3 \mathrm{a}$ & $68 \pm 12,6 \mathrm{a}$ & & $11,5 \pm 1,2 \mathrm{c}$ & $18,2 \pm 3,6 \mathrm{a}$ \\
35 & $100 \mathrm{a}$ & $47 \pm 8,6 \mathrm{~b}$ & & $11,1 \pm 0,3 \mathrm{c}$ & $15,8 \pm 1,3 \mathrm{a}$ \\
40 & $29 \pm 8,9 \mathrm{~b}$ & $3 \pm 2,0 \mathrm{c}$ & & $16,9 \pm 3,0 \mathrm{c}$ & - \\
$W$ & 0,4892 & 0,0048 & & 0,0907 & 0,599 \\
$F$ & 0,8124 & 0,0538 & & 0,1736 & 0,2720 \\
\hline
\end{tabular}

Barthlott 1995). A tolerância à seca e oscilações térmicas, apesar de não serem necessárias para seu estabelecimento, torna estas plantas dominantes em ambientes extremos (Alves \& Kolbek 1994). O padrão de germinação apresentado pelas sementes de Vellozia epidendroides e $V$. leptopetala, com alta germinabilidade em ampla faixa de temperatura, corrobora a sua adaptação ao ambiente rupestre, fato constatado por Garcia \& Diniz (2003) para outras espécies de Vellozia da Serra do Cipó (MG). As altas porcentagens de germinação apresentadas por sementes de espécies de Velloziaceae na faixa de 20 ${ }^{\circ} \mathrm{C}$ a $40{ }^{\circ} \mathrm{C}$, sob luz, em laboratório, indicam uma adaptação a áreas sujeitas a altas radiações solares e grandes flutuações circadianas de temperatura, como as descritas para campos de altitude (Menezes \& Giulietti 1986; Garcia \& Diniz 2003). Portanto, as sementes teriam a capacidade de tolerar e germinar sob as intensas variações diárias de temperatura do substrato.
As respostas germinativas são dependentes de uma combinação de condições ambientais, das quais a interação de luz e temperatura representa uma das mais complexas (Roberts 1981). Para as sementes de Vellozia leptopetala foi observado fotoblastismo restrito nas temperaturas mais baixas (faixa de 15 a $25^{\circ} \mathrm{C}$ ) e fotoblastismo relativo (sensu Ferreira et al. 2001) nas temperaturas mais altas $\left(30\right.$ e $\left.35^{\circ} \mathrm{C}\right)$. $V$. epidendroides apresentou fotoblastismo restrito apenas a $15{ }^{\circ} \mathrm{C}$ e, apesar de uma faixa mais ampla de fotoblastismo relativo, poucas sementes responderam ao tratamento de escuro na faixa de 20 a $30{ }^{\circ} \mathrm{C}$, com resposta significativa apenas a 35 e $40{ }^{\circ} \mathrm{C}$. Esta resposta diferenciada entre as espécies pode estar relacionada com o microhábitat que as mesmas ocupam. V. leptopetala estaria sujeita a maiores temperaturas e flutuações térmicas, por crescer sobre afloramentos rochosos, enquanto $V$. epidendroides teria a proteção de gramíneas cobrindo o substrato pedregoso.

Tabela 2. Germinabilidade final e tempo médio de germinação de sementes de Vellozia epidendroides Mart. ex Schult. \& Schult. (média \pm desvio padrão). Na coluna, valores seguidos por letras iguais não diferem estatisticamente entre si $(P>0,05)$. $W$ : estatística do teste de Shapiro-Wilk; $F$ : estatística do teste de Brown-Forsytle.

\begin{tabular}{|c|c|c|c|c|}
\hline \multirow[b]{2}{*}{ Temperatura $\left({ }^{\circ} \mathrm{C}\right)$} & \multicolumn{2}{|c|}{ Germinabilidade (\%) } & \multicolumn{2}{|c|}{ Tempo médio de germinação (dias) } \\
\hline & Luz & Escuro & Luz & Escuro \\
\hline $15^{\circ}$ & $0 \mathrm{~d}$ & $0 \mathrm{c}$ & - & - \\
\hline 20 & $29 \pm 6,8 \mathrm{c}$ & $3 \pm 3,8 \mathrm{bc}$ & $25,0 \pm 3,4 \mathrm{a}$ & - \\
\hline 25 & $60 \pm 7,3 b$ & $4 \pm 3,3 \mathrm{bc}$ & $12,1 \pm 0,8 b$ & - \\
\hline 30 & $91 \pm 3,8 \mathrm{a}$ & $14 \pm 7,7 \mathrm{~b}$ & $9,5 \pm 0,9 \mathrm{~b}$ & $9,1 \pm 1,1 \mathrm{~b}$ \\
\hline 35 & $93 \pm 6,0 \mathrm{a}$ & $73 \pm 10,5 a$ & $9,5 \pm 0,7 \mathrm{~b}$ & $9,9 \pm 0,5 \mathrm{~b}$ \\
\hline 40 & $84 \pm 14,2 a$ & $88 \pm 16,3 \mathrm{a}$ & $12,2 \pm 1,1 \mathrm{~b}$ & $12,4 \pm 1,2 \mathrm{a}$ \\
\hline$W$ & 0,3112 & 0,6156 & 0,0121 & 0,8703 \\
\hline$F$ & 0,1601 & 0,2274 & 0,3159 & 0,3405 \\
\hline
\end{tabular}


A fotodormência, observada em diferentes graus entre as sementes das espécies estudadas, pode desempenhar um papel importante na ocupação de uma área e na manutenção de uma população vegetal, por permitir a sobrevivência das sementes e a formação de um banco de sementes no solo (Pons 1991). A existência de um banco de sementes é especialmente importante para espécies que vivem em ambientes altamente variáveis ou imprevisíveis (Venable \& Brown 1988), como os campos rupestres, permitindo a recuperação de populações reduzidas ou mesmo dizimadas por eventos como queimadas e períodos secos prolongados. As sementes estocadas no solo, derivadas de diversos indivíduos, garantem a persistência de alelos presentes em seus progenitores no pool gênico da população (Fenner 1995).

A produção de sementes capazes de germinar em diferentes condições de luminosidade, como observado nas temperaturas mais altas, pode ser de grande valor para um indivíduo e para a população, por permitir a colonização de maior diversidade de hábitats como, por exemplo, fendas de rochas com pouca ou nenhuma luz, situação comum para Vellozia leptopetala, ou cobertura por herbáceas, como ocorre nos ambientes que $V$. epidendroides se estabelece.

\section{Agradecimentos}

Ao Dr. G.W. Fernandes, por permissão para desenvolver o estudo na sua propriedade; aos funcionários e diretor do Parque Nacional da Serra do Cipó, pelo apoio logístico; a Andréa Barbosa, pelo auxílio nos experimentos de germinação; à FAPEMIG pelo auxílio financeiro (proc. CRA 1590/98); aos assessores anônimos da Acta Botanica Brasilica, pela leitura criteriosa do manuscrito e contribuição significativa.

\section{Referências bibliográficas}

Abreu, M.E.P. \& Garcia, Q.S. 2005. Efeito da luz e da temperatura na germinação de sementes de quatro espécies de Xyris L. (Xyridaceae) ocorrentes na Serra do Cipó, MG, Brasil. Acta Botanica Brasilica 19: 149-154.

Alves R.J.V. \& Kolbek, J. 1994. Plant species endemism in savanna vegetation on table mountains (Campo Rupestre) in Brazil. Vegetatio 113: 125-139.

Baskin, C.C. \& Baskin, J.M. 1988. Germination ecophysiology of herbaceous plant species in a temperate region. American Journal of Botany 7(2): 286-305.
Fenner, M. 1995. Ecology of Seed Banks. Pp. 507-543. In: J. Kigel \& G. Galili (eds.). Seed development and germination. New York, Marcel Dekker.

Ferreira, A.G.; Cassol, B.; Rosa, S.G.T.; Silveira, T.S.; Stival, A.L. \& Silva, A.A. 2001. Germinação de sementes de Asteraceae nativas no Rio Grande do Sul, Brasil. Acta Botanica Brasilica 15: 231-242.

Franco, M. \& Silvertown, J. 1997. Life history variations in plants: an exploration of the fast-slow continuum hypothesis. Pp. 210-227. In: J. Silvertown; M. Franco \& J.L. Harper (eds.). Plant life histories. Cambridge, Cambridge University.

Gaff, D.F. 1987. Desiccation tolerant plants in South America. Oecologia 74: 133-136.

Garcia, Q.S. \& Diniz, I.S.S. 2003. Comportamento germinativo de três espécies de Velloziaceae dos campos rupestres de Minas Gerais. Acta Botanica Brasilica 17: 487-494.

Ghersa, C.M.; Beneth-Arnold, R.L. \& Martinez-Ghersa, M.A. 1992. The role of fluctuating temperatures in germination and establishment of Sorghum hapelense. Regulation of germination at increasing depths. Functional Ecology 6: $460-468$.

Giulietti, A.M.; Menezes, N.L.; Pirani, J.R.; Meguro, M. \& Wanderley, M.G. 1987. Flora da Serra do Cipó, Minas Gerais: caracterização e lista das espécies. Boletim de Botânica da Universidade de São Paulo 9: 1-152.

Giulietti, A.M. \& Pirani, J.R. 1988. Patterns of geographic distribution of some plant species from Espinhaço Range, Minas Gerais and Bahia, Brazil. Pp. 39-69. In: P.E. Vanzolini \& W. Ronald Heyer (eds.). Proceedings of a workshop on neotropical distribution on patterns. Rio de Janeiro, Academia Brasileira de Ciências.

Grime, J.P.; Mason, G.; Curtis, A.V.; Rodman, J.; Band, S.R.; Mowforth, M.A.G.; Neal, A.M. \& Shaw, S. 1981. A comparative study of germinaation characteristics in a local flora. Journal of Ecology 69: 1017-1059.

Haig, D. \& Westoby, M. 1988. On limits to seed production. American Naturalist 131: 757-759.

Jacobi, C.M. del Sarto, M.C.L. 2007. Pollination of two species of Vellozia (Velloziaceae) from high-altitude quartzitic grasslands, Brazil. Acta Botanica Brasilica 21: 325-333.

Labouriau, L.G. 1983. A germinação das sementes. Washington, D.C., Secretaria Geral da Organização dos Estados Americanos.

Leishmann, M.R.; Wright, I.J.; Moles, A.T. \& Westoby, M. 2000. The evolutionary ecology of seed size. Pp. 31-57. In: M. Fenner (ed.). The ecology of regeneration in plant communities. $2^{\text {nd }}$ ed. Wallingford, CABI International.

Mello-Silva, R. 1995. Aspectos taxonômicos, biogeográficos, morfológicos e biológicos das Velloziaceae de GrãoMogol, Minas Gerais, Brasil. Boletim de Botânica da Universidade de São Paulo 14: 49-79.

Mendonça, M.P. \& Lins, L.V. 2000. Lista vermelha das espécies ameaçadas de extinção da flora de Minas Gerais. Belo Horizonte, Fundação Biodiversitas \& Fundação Zoo-Botânica de Belo Horizonte. 
Menezes, N.L. \& Giulietti, A.M. 1986. Serra do Cipó - Paraíso dos Botânicos. Ciência Hoje 25(5): 38-44.

Moles, A.T.; Ackerly, D.D.; Webb. C.O.; Tweddle, J.C.; Dickie, J.B. \& Westoby, M. 2005. A brief history of seed size. Science 307: 576-580.

Nimer, E. 1989. Climatologia do Brasil. Rio de Janeiro, IBGE.

Oliveira, P.G. \& Garcia, Q.S. 2005. Efeitos da luz e da temperatura na germinação de sementes de Syngonanthus elegantulus Ruhland, S. elegans (Bong.) Ruhland e $S$. venusthus Silveira (Eriocaulaceae). Acta Botanica Brasilica 19: 639-645.

Owoseye, J.A. \& Sanford, W.W. 1972. Ecological study of Vellozia schnitzleinia, a drought-enduring plant of Northern Nigeria. Journal of Ecology 60(3): 807-817.

Pearson, T.R.H.; Burslem, F.R.D.; Mullins, C.E. \& Dalling, J.W. 2003. Functional significance of photoblastic germination in neotropical pioneer trees: a seed's eye view. Functional Ecology 17: 394-402.

Pons, T.L. 1991. Induction of dark dormancy in seeds: its importance for the seed bank in the soil. Functional Ecology 5: 669-675.

Porembski, S. \& Barthlott, W. 1995. On the occurrence of a velamen radicum in Cyperaceae and Velloziaceae. Nordic Journal of Botany 15(6): 625-629.

Porembski, S. \& Barthlott, W. 2000. Granitic and gneissic outcrops (inselbergs) as centers of diversity for desiccation-tolerant vascular plants. Plant Ecology 151: 19-28.
Roberts, E. H. 1981. The interaction of environmental factors controlling loss of dormancy in seeds. Annals of Applied Biology 98(3): 552-555.

Sazima, M. \& Sazima, I. 1990. Hummingbird pollination in two species of Vellozia (Liliiflorae, Velloziaceae) in southeastern Brazil. Botanica Acta 103: 83-86.

Sendulsky, T. \& Burman, A.G. 1978. Paspalum species of Serra do Cipó (1): a contribution to the study of the Brazilian Poaceae. Revista Brasileira de Botânica 1(1): 1-15.

Smith, L.B. \& Ayensu, E.S. 1976. A revision of American Velloziaceae. Smithsonian Contributions to Botany 30: $1-172$.

Stephenson, A.G. 1981. Flower and fruit abortion: proximate causes and ultimate functions. Annual Review of Ecology and Systematics 12: 253-279.

Thompson, K.; Band, S.R. \& Hodgson, J.G. 1993. Seed size and shape predict persistence in soil. Functional Ecology 67: 893-921.

Vázquez-Yanes, C. \& Orozco-Segovia, A. 1993. Patterns of seed longevity and germination in the tropical rain forest. Annual Review in Ecology and Systematics 24: 69-87.

Venable, D.L. \& Brown, J.S. 1988. The selective interactions of dispersal, dormancy and seed size as adaptations for reducing risk in variable environments. American Naturalist 131: 360-383.

Wulff, R.D. 1995. Environmental maternal effects on seed quality and germination. Pp. 491-505. In: J. Kigel \& G. Galili (eds.). Seed development and germination. New York, Marcel Dekker. 